\title{
The efficacy of serum copeptin and zinc-alpha2-glycoprotein for predictors of tolvaptan treatment in decompensated cirrhotic patients
}

Ryuta Shigefuku ( $\nabla$ shigefuku@clin.medic.mie-u.ac.jp)

Mie University Graduate School ofMedicine https://orcid.org/0000-0002-6738-4382

Motoh Iwasa

Mie University Graduate School of Medicine

Akiko Eguchi

Mie University Graduate School of Medicine

Mina Tempaku

Mie University School of Medicine

Yasuyuki Tamai

Mie University School of Medicine

Tatsuya Suzuki

Mie University Graduate School of Medicine

Yoshiyuki Takei

Mie University Graduate School of Medicine

\section{Research article}

Keywords: copeptin, zinc-a2-glycoprotein (ZAG), tolvaptan, liver cirrhosis, ascites

Posted Date: August 12th, 2020

DOI: https://doi.org/10.21203/rs.3.rs-52252/v1

License: @ (1) This work is licensed under a Creative Commons Attribution 4.0 International License. Read Full License 


\section{Abstract}

\section{Aim}

The efficacy of tolvaptan, an orally active vasopressin V2-receptor antagonist, has recently been reported in patients with refractory ascites unresponsive to conventional diuretics. However, the effect of tolvaptan varies among patients. Recently, the prognostic role of tolvaptan response in decompensated liver cirrhosis (LC) is also attracts attention. Our aim is to elucidate predictive factors using serum copeptin (vasopressin precursor), zinc-a2-glycoprotein (ZAG), cystatin C (renal biomarker), neutrophil gelatinase-associated lipocalin (NGAL) and liver-type fatty acid-binding protein (L-FABP) that portend a good response to tolvaptan in LC patients with ascites.

\section{Methods}

We enrolled 113 LC patients and divided into tolvaptan treatment group and non-treatment group. Tolvaptan (3.75 or $7.5 \mathrm{mg} / \mathrm{day})$ was administrated to $38 \mathrm{LC}$ patients with ascites and a follow-up assessment occurred after a 7-day tolvaptan treatment regimen.

\section{Results}

We revealed predictive ability for kidney and/or liver damage in serum copeptin, ZAG, cystatin C, NGAL and L-FABP levels in all patients. Post 7-day tolvaptan treatment, 19 patients lost more than $1.5 \mathrm{~kg}$ of their body weight (Responders), while 19 patients did not change their body weight significantly (Non-responders). Basal blood urea nitrogen (BUN) levels $(p=0.0014)$, serum copeptin ( $p$ $=0.0265)$ and serum ZAG levels $(p=0.0142)$ were significantly higher in the Non-responders. BUN (odds ratio 7.43, $p=0.0306$ ), copeptin (odds ratio 9.12, $p=0.0136$ ) and ZAG (odds ratio 7.43, $p=0.0306$ ) were determined to be predictive factors of drug responsiveness using multivariate logistic regression analysis.

\section{Conclusion}

Serum BUN, copeptin and ZAG levels predict patient response to tolvaptan even when measured prior to treatment.

\section{Introduction}

Arginine vasopressin (AVP) is a potent antidiuretic hormone in the human body. Despite the clinical relevance of AVP in maintaining fluid balance and vascular tone, measurement of the mature form of AVP is difficult due to small size ( 9 amino acids), short half-life, and its ability to bind platelets ${ }^{1}$. Copeptin, a 39 -amino acid glycopeptide that comprises the C-terminal part of the AVP precursor, was found to be a stable and sensitive surrogate marker for AVP release ${ }^{2}$. Copeptin would be particularly helpful in diseases whereby primary or secondary (e.g. cardiovascular instability, chronic heart failure, sepsis) disturbances of the vasopressinergic system contribute to disease pathogenesis ${ }^{3}$. Current reports have demonstrated that a high serum copeptin concentration predicts survival in a heterogeneous population of hospitalized liver cirrhosis (LC) patients, independent of liver-specific scoring systems ${ }^{4}$, suggesting that copeptin has the potential to be a biomarker of disease progression and prognosis in LC 5 .

Zinc-alpha-2-glycoperotein (ZAG) is a 41-kDa glycoprotein assigned to the major histocompatibility complex (MHC) class I family of proteins. ZAG is a secretory protein synthesized by epithelial cells and adipocytes and plays a role in lipid metabolism, cell cycling and cancer progression. ZAG has been reported as a biomarker of renal injury based on microarray analyses showing an increase of ZAG expression in aged proximal tubular cells ${ }^{6}$. In addition, a clinical investigation showed serum ZAG levels can predict mortality in hemodialysis patients ${ }^{7}$. However, these changes in serum ZAG levels have not been reported in LC patients with refractory ascites.

There are several established markers of renal function, such as serum cystatin C, neutrophil gelatinase-associated lipocalin (NGAL), and liver-type fatty acid binding protein (L-FABP). Serum cystatin $C$ is a cysteine protease inhibitor produced in nearly all nucleated cells. As opposed to creatinine, cystatin $\mathrm{C}$ is not influenced by muscle mass, thus permitting an accurate evaluation of renal function 
even in patients with low muscle mass. Urea NGAL is a binding partner with matrix metalloproteinase-9 in neutrophils ${ }^{8}$ and is established as a clinical biomarker for acute renal injury ${ }^{9}$ based on NGAL function of a kidney protector ${ }^{10}$. Urine L-FABP levels derived from proximal tubular epithelial cells are elevated in renal tubular injury episodes and are therefore used as an established marker of several kidney diseases, including acute kidney injury and chronic kidney diseases ${ }^{12}$. The primarily expression of NGAL and L-FABP in the liver led us to investigate whether serum NGAL and serum L-FABP reflect liver and kidney function. We have reported that serum NGAL and serum L-FABP is are prognostic factors for survival in chronic liver diseases ${ }^{11,13}$.

Ascites is the most frequent complication of $L C$ and refractory ascites has a negative prognostic implication in the natural progression of LC. Tolvaptan is a novel, nonpeptide, orally administered, selective vasopressin $\mathrm{V}_{2}$-receptor antagonist that downregulates the expression of aquaporin-2 in the renal collecting duct. Current research has uncovered a promising role for tolvaptan as an add-on treatment in patients with hepatic ascites resistant to furosemide and/or spironolactone, as it is able to decrease body weight and alleviate edema ${ }^{14,15}$. However, the effect of tolvaptan is highly variable among patients with approximately one half of the patient cohort responding to the drug ${ }^{14}$. Blood urea nitrogen (BUN) ${ }^{14}$ and C-reactive protein (CRP) ${ }^{15}$ are recognized for differentiating diagnoses between Responders and Non-responders for tolvaptan, but these factors are insufficient, thus additional biomarkers are needed. Recently, in addition to the aforementioned studies, several studies have also reported Responders to tolvaptan led to improvement of long-term survival rates in cirrhotic patients with ascites ${ }^{16,17}$. Therefore, the aim of this study is to assess the correlation between the efficacy of tolvaptan and treatment related factors, including serum copeptin, ZAG, cystatin C, NGAL and L-FABP levels in LC patients with ascites.

\section{Patients And Methods}

\section{Human samples}

The study protocol was approved by the Clinical Research Ethics Review Committee of Mie University Hospital. This study was performed retrospectively on stored samples, and patients could opt out of their data. In this study, we enrolled 113 LC patients (69 males and 44 females) with a mean age of $67.5 \pm 10.9$ years who could be analyzed between November 2013 and September 2016 at Mie University hospital. We divided the 113 LC patients into tolvaptan treatment group or non-treatment group. The tolvaptan treatment group was consisted of 45 patients. LC was diagnosed based on morphologic changes of the liver such as hypertrophy of the left lateral and caudate lobes, or atrophy of the right posterior hepatic lobe on ultrasonography and through blood tests, and/or computed tomography, magnetic resonance imaging, FibroScan (Echosens, French) results, and esophageal varix by endoscopy, as is the general protocol. HCC was diagnosed based on histological findings or typical imaging characteristics. Body mass index (BMI) was calculated as weight $(\mathrm{kg}) /$ height $(\mathrm{m})$ squared. Using cross-sectional CT at the level of transverse process of lumbar vertebra L3, the bilateral psoas muscle area was identified. Psoas muscle index (PMI) $\left(\mathrm{cm}^{2} / \mathrm{m}^{2}\right)$ was defined by normalizing psoas muscle area $\left(\mathrm{cm}^{2}\right) /$ height $(\mathrm{m})$ squared. Patients who had other malignancies within the past 3 years, spontaneous bacterial peritonitis, hepatic encephalopathy (coma scale score $\geq \mathrm{II}$ ), heart failure (the New York Heart Association defined category $\geq$ class II), human immunodeficiency virus infection, pregnancy, or psychiatric problems were deemed to be unsuitable for clinical study.

\section{Serum preparation}

Serum samples were collected when patients showed up at the hospital or on the same day that tolvaptan was administered, and albumin $(\mathrm{g} / \mathrm{dL})$, total bilirubin $(\mathrm{mg} / \mathrm{dL})$, sodium $(\mathrm{mEq} / \mathrm{L})$, creatinine $(\mathrm{mg} / \mathrm{dL}), B U N(\mathrm{mg} / \mathrm{dL})$ and $\mathrm{CRP}(\mathrm{mg} / \mathrm{dL})$ were measured. Serum were kept at $-80^{\circ} \mathrm{C}$ until copeptin $(\mathrm{pmol} / \mathrm{L}), \mathrm{ZAG}(\mu \mathrm{g} / \mathrm{dL})$, cystatin $\mathrm{C}(\mathrm{mg} / \mathrm{dL}), \mathrm{NGAL}(\mathrm{ng} / \mathrm{mL})$ and L-FABP $(\mathrm{ng} / \mathrm{mL}) \mathrm{measurements}$, using an automated copeptin immunofluorescent assay kit (Thermo Fisher Scientific Inc., Tokyo) ${ }^{2}$, ZAG enzyme-linked immunosorbent assay (ELISA) kit (BioVendor, Czech Republic), cystatin C ELISA kit (R\&D systems, Minneapolis, MN), NGAL ELISA kit (R\&D systems, Minneapolis, MN) and high-sensitivity human L-FABP ELISA kit (CMIC Holdings Co., Ltd., Tokyo), respectively. The Child-Pugh score, albumin-bilirubin (ALBI) score ${ }^{18}$, the fibrosis index based on 4 factors (FIB-4) ${ }^{19}$, model for end-stage liver disease (MELD) score and estimated glomerular filtration rate (eGFR) were calculated.

\section{Statistical analysis}

All data are expressed as mean \pm SD. Data were analyzed using the Mann-Whitney $U$ test in two groups and one-way analysis of variance for comparison of continuous variables. The relationship between serum copeptin, ZAG, cystatin C, NGAL, L-FABP levels 
and clinical data were examined using Spearman's rank correlation coefficient. For each continuous variable, the optimal cutoff value that maximized the sum of sensitivity and specificity was selected using receiver operating characteristic (ROC) analysis for survival. A logistic regression analysis was utilized for the multivariate analysis in order to evaluate the relationship between effect of tolvaptan and clinical data. Only variables deemed to be significant $(p<0.1)$ in the univariate analysis were included in the subsequent multivariate analysis. The statistical analyses were performed using JMP software program (SAS Institute, Cary, NC, USA) for univariate and multivariate logistic regression analysis. Differences were considered to be significant at $p<0.05$.

\section{Results}

\section{Clinical characteristics of patients with or without tolvaptan}

In this study, we divided the 113 LC patients into tolvaptan treatment group or non-treatment group. Table 1 shows the comparison of baseline clinical characteristics and laboratory variables between patients with and without tolvaptan treatment. In the tolvaptan treatment group, dose of diuretic drugs, the Child-Pugh score, ALBI score, and serum copeptin, NGAL and L-FABP levels were significantly increased, while BMI, PMI, serum albumin levels were reduced, suggesting that patients treated with tolvaptan demonstrated more advanced liver diseases. In contrast, there were no obvious differences in age, gender, prevalence of HCC, creatinine, BUN, eGFR, ZAG and cystatin C between the two groups (Table 1).

\section{Correlation of copeptin, ZAG, cystatin C, NGAL and L-FABP to clinical parameters in all patients}

The correlations between copeptin, ZAG, cystatin C, NGAL or L-FABP and clinical parameters in LC patients are shown in Table 2. Copeptin was strongly correlated with mainly hepatic function including albumin ( $p=0.007$; Figure 1-D), Child-Pugh score $(p<0.001)$, ALBI score $(p=0.0003)$ and CRP $(p=0.0047 ;$ Figure $1-C)$. In contrast, ZAG was more strongly correlated with renal function including creatinine $(p<0.0001), B U N(p=0.0001)$ and eGFR ( $p=0.001)$ (Table 2; Figure 1-K and $L)$. In addition, ZAG was correlated with bodyweight $(p=0.0092$; Figure $1-G)$ and BMI $(p=0.0001$; Figure 1-H) and CRP $(p<0.0001$; Figure 1-I). Cystatin C was significantly correlated with age $(p=0.0191)$, renal parameters (creatinine and BUN: $p<0.0001$ and eGFR: $p=0.001)$, copeptin $(p=0.008), N G A L$ $(p<0.0001)$ and L-FABP $(p<0.0001)$ (Table 2). NGAL and L-FABP were significantly correlated with indicators of both renal function and hepatic function (Table 2). MELD score significantly correlated with all, copeptin ( $p=0.001), Z A G(p=0.0003)$, cystatin $C$ $(p<0.0001)$, NGAL $(p<0.0001)$ and L-FABP $(p=0.0024)$.

\section{Background comparison between Responders and Non-responders to tolvaptan}

In this study, we excluded 7 from 45 cases for which tolvaptan efficacy could not be determined because of transferring hospitals, lack of weight data or with other treatment such as albumin transfusion, ascites puncture, and cell-free and concentrated ascites reinfusion therapy (Supplementary Figure 1). We enrolled 38 decompensated LC patients with ascites (24 males and 14 females) with a mean age of $67.1 \pm 9.7$ years. We divided the 38 patients into two tolvaptan treatment groups: Responders and Nonresponders. The Non-responder group was defined as patients with weight loss of $<1.5 \mathrm{~kg} /$ week after receiving tolvaptan or performing paracentesis within the first week ${ }^{12}$. All patients continued to take the same prescribed doses of furosemide and spironolactone within the first week. There were no obvious differences in age, gender, bodyweight, BMI, PMI, presence of HCC, dose of diuretic drugs, albumin, total bilirubin, Child-Pugh score, ALBI score, FIB4-index, MELD score, creatinine, eGFR, serum sodium, cystatin C, NGAL and L-FABP between the two groups (Table 3; Figure 2-D, E and F). In contrast, BUN, copeptin and ZAG levels were significantly higher in Non-responders when compared to the Responders group (respectively, $p=0.0014, p=0.0265, p=0.0142)(T a b l e$ 3; Figure 2-A, B and C). We calculated the cutoff values, area under the ROC curve, sensitivity and specificity of BUN, copeptin and ZAG using ROC analysis. The cutoff values ascertained from our analyses of BUN, copeptin and ZAG were $18.3 \mathrm{mg} / \mathrm{dL}, 10.1 \mathrm{pmol} / \mathrm{L}$ and $32.4 \mu \mathrm{g} / \mathrm{mL}$, respectively (Table 3 ).

\section{Predictors contributing to the effect of tolvaptan in treatment for ascites}

Using multivariate logistic regression analysis, we found BUN (odds ratio 7.43, $p=0.0306$ ), serum copeptin levels (odds ratio 9.12 , $p=0.013$ ) and ZAG (odds ratio $7.43, p=0.0306$ ) to be the significant predictors contributing to the efficacy of tolvaptan in the treatment for ascites (Table 4).

\section{Discussion}


In this study, we revealed for the first time that serum copeptin and ZAG levels were significantly increased in the tolvaptan treatment Non-responders group when compared to the Responders group. In addition, we indicated that serum BUN, copeptin and ZAG levels were independent predictors of the overall response to tolvaptan therapy. We further showed that serum creatinine and cystatin $C$ levels were not predictive of patient response to tolvaptan, thus confirming the findings reported by others ${ }^{14,15}$. In LC, arteriolar vasodilation causes underfilling of the systemic arterial vascular space, and the decrease in the effective blood volume leads to a decrease in arterial pressure ${ }^{21}$. Consequently, activation of the renin-angiotensin-aldosterone system, sympathetic nervous system and the release of antidiuretic hormone take place as the body attempts to restore normal blood pressure homeostasis ${ }^{22}$. The main physiological function of AVP is antidiuresis, thereby regulating systemic osmotic pressure. Plasma AVP levels are normally regulated by plasma osmotic pressure and have been found to be elevated in LC patients with ascites, as reported by Pérez-Ayuso $\mathrm{RM}^{23}$. However, there has been no study to date exploring whether the effect of tolvaptan is related to plasma AVP or pro-AVP (copeptin) levels in ascites patients. The Non-responder group is considered to be in a state of relative vascular underfilling with an increase in BUN and copeptin, thus suggesting intravascular dehydration ${ }^{14}$. Furthermore, serum copeptin also correlated with multiple factors including renal function and CRP, resulting in copeptin is not only a biomarker of renal function. Although NGAL and L-FABP are useful markers than copeptin for acute-on-chronic liver failure ${ }^{24}$ and acute kidney injury ${ }^{25}$, respectively, this study indicated that serum NGAL and L-FABP were not useful in assessing the efficacy of response to tolvaptan in LC patients with ascites.

Serum ZAG, it has been determined, is increased in acute kidney injury ${ }^{26}$ and in minor kidney injury caused by normo-albuminuric diabetic kidney disease - patients presenting with renal insufficiency, but no significant proteinuria ${ }^{27}$. On the other hand, serum cystatin $\mathrm{C}$ accurately reflects renal function, while ZAG reflects lipolysis and renal function. Serum ZAG has been expected as a biomarker for cachexia ${ }^{28}$. Indeed, in this study, ZAG correlated with body weight, BMI and CRP. Bellos also reported that body weight loss and elevated CRP were the predictors for Non-responders to tolvaptan ${ }^{15}$. We assumed that association of serum ZAG with multiple factors including renal function, cachexia (relating to body weight and $\mathrm{BMI}$ ) and/or inflammation (relating to CRP) led to the result.

Based on the evidence outlined above, serum copeptin may reveal the underlying pathological condition of decreased osmotic pressure, intravascular dehydration and inflammation, whereas serum ZAG may reflect minor kidney injury, cachexia and inflammation, suggesting that copeptin and ZAG are independent predictors of patient response to tolvaptan.

This study has several limitations. This was a retrospective and single-center study with a small sample size and a short follow-up period. Further study using a larger cohort is required to investigate the interaction of copeptin, ZAG, and BUN and the possible predictors of patient survival. In conclusion, serum BUN $>18.3 \mathrm{mg} / \mathrm{dL}$, copeptin $<10.1 \mathrm{pmol} / \mathrm{L}$ and ZAG levels of $<32.4 \mu \mathrm{g} / \mathrm{ml}$ appear to be good predictors of overall patient response to tolvaptan treatment.

\section{Conclusions}

Serum copeptin, ZAG and BUN levels can be used as novel biomarkers to determine overall response to tolvaptan in patients presenting with LC and ascites. Future study is required to develop a new clinical prediction model using serum copeptin, ZGA and BUN that would help clinicians determine the effectiveness of tolvaptan treatment.

\section{Declarations}

\section{Acknowledgement}

There was no acknowledgement.

\section{Statement of Ethics}

The study protocol was approved by the Clinical Research Ethics Review Committee of Mie University Hospital (approval no. H2019190). This research comply with the guidelines for human studies and should include evidence that the research was conducted ethically in accordance with the World Medical Association Declaration of Helsinki. The subjects (or their parents or legal guardians) have given their written informed consent. 
Authors does not have any conflict of interests, except Motoh Iwasa received a lecture fee from Otsuka Pharmaceutical Co., Ltd, which manufactures tolvaptan.

\section{Funding Sources}

No external financial support was received.

\section{Author Contributions}

RS: statistical analysis, data interpretation and drafting the article; MI: study concept, design, data acquisition, data interpretation and drafting the article; $\mathrm{AE}$ : sample analyses, data interpretation and critical revision of the article for important intellectual content; MT: sample analyses; YT: sample collection; TS: data analysis; YT: critical revision of the article for important intellectual content; All authors: approval of the final version of the manuscript

\section{Abbreviations}

LC, liver cirrhosis; ZAG, zinc-a2-glycoprotein; AVP, arginine vasopressin; NGAL, neutrophil gelatinase-associated lipocalin; L-FABP, liver-type fatty acid-binding protein; BMI, Body mass index, PMI, Psoas muscle index; BUN, blood urea nitrogen; ALBI, albuminbilirubin; FIB-4, fibrosis index based on 4 factors; MELD, Model for end-stage liver disease; eGFR, estimated glomerular filtration rate; $\mathrm{ROC}$, receiver operating characteristic

\section{References}

1. Bolignano D, Cabassi A, Fiaccadori E, Ghigo E, Pasquali R, Peracino A, et al. Copeptin (CTproAVP), a new tool for understanding the role of vasopressin in pathophysiology. Clin Chem Lab Med. 2014;52(10):1447-56.

2. Morgenthaler NG, Struck J, Alonso C, Bergmann A. Assay for the measurement of copeptin, a stable peptide derived from the precursor of vasopressin. Clin Chem. 2006;52(1):112-9.

3. Morgenthaler NG, Struck J, Jochberger S, Dunser MW. Copeptin: clinical use of a new biomarker. Trends Endocrin Met. 2008;19(2):43-9.

4. Kerbert AJC, Weil D, Verspaget HW, Moréno JP, Hoek BV, Cervoni JP, et al. Copeptin is an independent prognostic factor for transplant-free survival in cirrhosis. Liver Int. 2016;36(4):530-7.

5. Solà E, Kerbert AJC, Verspaget HW, Moreira R, Pose E, Ruiz P, et al. Plasma copeptin as biomarker of disease progression and prognosis in cirrhosis. J Hepatol. 2016;65(5):914-20.

6. Schmitt R, Marlier A, Cantley LG. Zag expression during aging suppresses proliferation after kidney injury. J Am Soc Nephrol. 2008;19(12):2375-83.

7. Bouchara A, Yi D, Pastural M, Granjon S, Selag JC, Laville M, et al. Serum levels of the adipokine zinc-alpha2-glycoprotein (ZAG) predict mortality in hemodialysis patients. Kidney Int. 2018;94(5):983-92.

8. Kjeldsen $L$, Johnsen $A H$, Sengelov $H$, Borregaard N. Isolation and primary structure of NGAL, a novel protein associated with human neutrophil gelatinase. J Biol Chem. 1993;268(14):10425-32.

9. Mishra J, Dent C, Tarabishi R, Mitsnefes MM, Ma Q, Kelly C, et al. Neutrophil gelatinase-associated lipocalin (NGAL) as a biomarker for acute renal injury after cardiac surgery. Lancet. 2005;365(9466):1231-8.

10. Mori K, Lee HT, Rapoport D, Drexler IR, Foster K, Yang J, et al. Endocytic delivery of lipocalin-siderophore-iron complex rescues the kidney from ischemia-reperfusion injury. J Clin Invest. 2005;115(3):610-21.

11. Yoshikawa K, Iwasa M, Eguchi A, Kojima S, Yoshizawa N, Tempaku M, et al. Neutrophil gelatinase-associated lipocalin level is a prognostic factor for survival in rat and human chronic liver diseases. Hepatol Commun. 2017;1(9):946-56.

12. Ichikawa D, Kamijo-lkemori A, Sugaya T, Ohata K, Hisamichi M, Hoshino S, et al. Utility of urinary tubular markers for monitoring chronic tubulointerstitial injury after ischemia-reperfusion. Nephrology (Carlton). 2018;23(4):308-16. 
13. Eguchi A, Hasegawa H, Iwasa M, Tamai Y, Ohata K, Oikawa T, et al. Serum Liver-Type Fatty Acid-Binding Protein Is a Possible Prognostic Factor in Human Chronic Liver Diseases From Chronic Hepatitis to Liver Cirrhosis and Hepatocellular Carcinoma. Hepatol Commun. 2019;3(6):825-37.

14. Kawaratani H, Fukui H, Moriya K, Noguchi R, Namisaki T, Uejima M, et al. Predictive parameter of tolvaptan effectiveness in cirrhotic ascites. Hepatol Res. 2017;47(9):854-61.

15. Bellos I, Kontzoglou K, Perrea DN. Predictors of tolvaptan short-term response in patients with refractory ascites: A metaanalysis. J Gastroenterol Hepatol. 2020;35(2):182-91.

16. Atsukawa M, Tsubota A, Takaguchi K, Toyoda H, Iwasa M, Ikegami T, et al. Analysis of factors associated with the prognosis of cirrhotic patients who were treated with tolvaptan for hepatic edema. J Gastroenterol Hepatol. 2020;35(7):1229-37.

17. Bellos I, Kontzoglou K, Psyrri A, Pergialiotis V. Tolvaptan Response Improves Overall Survival in Patients with Refractory Ascites: A Meta-Analysis. Dig Dis. 2020;38(4):320-8.

18. Johnson PJ, Berhane S, Kagebayashi C, Satomura S, Teng M, Reeves HL, et al. Assessment of liver function in patients with hepatocellular carcinoma: a new evidence-based approach-the ALBI grade. J Clin Oncol. 2015;33(6):550-8.

19. Sterling RK, Lissen E, Clumeck N, Sola R, Correa MC, Montaner J, et al. Development of a simple noninvasive index to predict significant fibrosis in patients with HIV/HCV coinfection. Hepatology. 2006;43(6):1317-25.

20. Hiramine $Y$, Uojima H, Nakanishi H, Hiramatsu A, Iwamoto T, Kimura M, et al. Response criteria of tolvaptan for the treatment of hepatic edema. J Gastroenterol. 2018;53(2):258-68.

21. Moller S, Bendtsen F. The pathophysiology of arterial vasodilatation and hyperdynamic circulation in cirrhosis. Liver Int. 2018;38(4):570-80.

22. Schrier RW, Arroyo V, Bernardi M, Epstein M, Henriksen JH, Rodes J. Peripheral arterial vasodilation hypothesis: a proposal for the initiation of renal sodium and water retention in cirrhosis. Hepatology. 1988;8(5):1151-7.

23. Zhao R, Wu W, Zhou Z, Zheng X, Sun W, Shi Y, et al. Prognostic utility of novel biomarkers in acute-on-chronic liver failure (ACLF) associated with hepatitis B: A multicenter prospective study. Hepatol Res. 2019;49(1):42-50.

24. Belcher JM, Parikh CR, Garcia-Tsao G. Acute kidney injury in patients with cirrhosis: perils and promise. Clin Gastroenterol Hepatol. 2013;11(12):1550-8.

25. Pérez-Ayuso RM, Arroyo V, Camps J, Rimola A, Gaya J, Costa J, et al. Evidence that renal prostaglandins are involved in renal water metabolism in cirrhosis. Kidney Int. 1984;26(1):72-80.

26. Sorensen-Zender I, Beneke J, Schmidt BM, Menne J, Haller H, Schmitt R. Zinc-alpha2-glycoprotein in patients with acute and chronic kidney disease. BMC Nephrol. 2013;14:145.

27. Lim SC, Liying DQ, Toy WC, Wong M, Yeoh LY, Tan C,et al. Adipocytokine zinc alpha2 glycoprotein (ZAG) as a novel urinary biomarker for normo-albuminuric diabetic nephropathy. Diabet Med. 2012;29(7):945-9.

28. Bing C, Trayhurn P. New insights into adipose tissue atrophy in cancer cachexia. Proc Nutr Soc. 2009;68:385-92.

\section{Tables}

Table 1. Characteristics of 113 liver cirrhotic patients. 


\begin{tabular}{|c|c|c|c|c|}
\hline \multirow[t]{2}{*}{ Parameters } & All & Non-treatment & Treatment & $p^{+}$ \\
\hline & $\mathrm{n}=113$ & $\mathrm{n}=68$ & $\mathrm{n}=45$ & \\
\hline Age (years) & $67.5 \pm 10.9$ & $67.2 \pm 11.6$ & $68.1 \pm 9.6$ & 0.9368 \\
\hline Gender, Male : Female & $69: 44$ & $43: 25$ & $26: 19$ & 0.6158 \\
\hline Bodyweight (kg) & $59.5 \pm 10.9$ & $61.2 \pm 11.2$ & $57.2 \pm 9.9$ & 0.1253 \\
\hline Body mass index $\left(\mathrm{kg} / \mathrm{m}^{2}\right)$ & $23.2 \pm 3.6$ & $23.8 \pm 3.4$ & $22.5 \pm 3.7$ & 0.0476 \\
\hline Psoas muscle index $\left(\mathrm{cm}^{2} / \mathrm{m}^{2}\right)$ & $5.7 \pm 1.7$ & $6.0 \pm 1.9$ & $5.4 \pm 1.3$ & 0.0434 \\
\hline $\mathrm{HCV} / \mathrm{HBV} / \mathrm{HCV}+\mathrm{AL} / \mathrm{AL} / \mathrm{NBNC} /$ others & $56 / 7 / 4 / 23 / 13 / 10$ & $39 / 3 / 1 / 13 / 6 / 6$ & $17 / 4 / 3 / 10 / 7 / 4$ & - \\
\hline presence of HCC, yes: no & $61: 52$ & $33: 35$ & $28: 17$ & 0.1970 \\
\hline \multicolumn{5}{|l|}{ Dose of diuretic drugs } \\
\hline Tolvaptan (3.75 mg/7.5 mg) & $37 / 8$ & $0 / 0$ & $37 / 8$ & - \\
\hline Furosemide (mg) & $13.3 \pm 19.0$ & $4.8 \pm 13.3$ & $26.4 \pm 19.0$ & $<0.0001$ \\
\hline Spironolactone (mg) & $21.7 \pm 25.9$ & $10.3 \pm 22.0$ & $38.9 \pm 21.4$ & $<0.0001$ \\
\hline AST (U/L) & $57.2 \pm 39.3$ & $47.4 \pm 29.4$ & $72.1 \pm 47.0$ & 0.0004 \\
\hline ALT (U/L) & $35.4 \pm 26.4$ & $33.9 \pm 26.3$ & $37.6 \pm 26.3$ & 0.2200 \\
\hline Albumin (g/dL) & $3.3 \pm 0.6$ & $3.5 \pm 0.6$ & $2.9 \pm 0.5$ & $<0.0001$ \\
\hline Total Bilirubin (mg/dL) & $1.7 \pm 2.1$ & $1.2 \pm 1.2$ & $2.4 \pm 3.1$ & 0.0012 \\
\hline Prothrombin time & $71.1 \pm 18.7$ & $74.2 \pm 16.3$ & $66.5 \pm 20.9$ & 0.0153 \\
\hline Child-Pugh score & $7.5 \pm 2.0$ & $6.5 \pm 1.5$ & $9.0 \pm 1.8$ & $<0.0001$ \\
\hline ALBI score & $-1.9 \pm 0.6$ & $-2.2 \pm 0.5$ & $-1.5 \pm 0.5$ & $<0.0001$ \\
\hline FIB4-index & $7.3 \pm 4.4$ & $6.2 \pm 3.8$ & $8.9 \pm 4.7$ & 0.0011 \\
\hline MELD score & $17.4 \pm 7.0$ & $17.4 \pm 8.6$ & $17.5 \pm 3.4$ & 0.0264 \\
\hline Creatinine (mg/dL) & $0.9 \pm 0.7$ & $1.0 \pm 0.9$ & $0.9 \pm 0.4$ & 0.3419 \\
\hline BUN (mg/dL) & $17.5 \pm 7.7$ & $16.5 \pm 7.4$ & $19.1 \pm 7.9$ & 0.0946 \\
\hline eGFR (mL/min/1.73m²) & $69.1 \pm 26.8$ & $70.4 \pm 26.0$ & $67.3 \pm 27.7$ & 0.2824 \\
\hline Serum sodium (mEq/L) & $137.2 \pm 4.5$ & $139.2 \pm 3.2$ & $134.5 \pm 4.7$ & $<0.0001$ \\
\hline CRP (mg/dL) & $1.2 \pm 2.1$ & $0.3 \pm 0.5$ & $2.4 \pm 2.8$ & $<0.0001$ \\
\hline Copeptin (pmol/L) & $12.7 \pm 18.4$ & $9.3 \pm 15.2$ & $17.9 \pm 21.4$ & 0.0007 \\
\hline $\mathrm{ZAG}(\mu \mathrm{g} / \mathrm{mL})$ & $31.8 \pm 10.9$ & $31.1 \pm 10.5$ & $32.8 \pm 11.4$ & 0.4251 \\
\hline NGAL (ng/mL) & $87.2 \pm 94.1$ & $67.6 \pm 87.2$ & $116.9 \pm 96.3$ & $<0.0001$ \\
\hline Cystatin C (mg/dL) & $1.1 \pm 0.4$ & $1.1 \pm 0.4$ & $1.2 \pm 0.4$ & 0.4099 \\
\hline L-FABP $(\mathrm{ng} / \mathrm{mL})$ & $10.7 \pm 10.2$ & $9.0 \pm 9.7$ & $13.1 \pm 10.5$ & 0.0124 \\
\hline
\end{tabular}

${ }^{\dagger}$ Mann-Whitney Utest (Non-treatment vs Treatment). HCV, hepatitis C virus; HBV, hepatitis B virus; AL, alcohol; NBNC, non HCV non HBV; HCC, hepatocellular carcinoma; AST, aspartate aminotransferase; ALT, alanine aminotransferase; ALBI, albumin-bilirubin; FIB4index, fibrosis-4; MELD, Model for End-Stage Liver Disease; BUN, blood urea nitrogen; eGFR, estimated glomerular filtration rate; CRP, C-reactive protein; ZAG, zinc-a2-glycoprotein; NGAL, neutrophil gelatinase-associated lipocalin; L-FABP, Liver-type fatty acid-binding protein. 
Table 2. Correlation between serum copeptin, ZAG, cystatin C, NGAL, L-FABP levels and clinical parameters in 113 liver cirrhotic patients.

\begin{tabular}{|c|c|c|c|c|c|c|c|c|c|c|}
\hline \multirow[t]{2}{*}{ Parameter } & \multicolumn{2}{|l|}{ Copeptin } & \multicolumn{2}{|l|}{ ZAG } & \multicolumn{2}{|c|}{ Cystatin C } & \multicolumn{2}{|l|}{ NGAL } & \multicolumn{2}{|l|}{ L-FABP } \\
\hline & $r$ & $p^{*}$ & $r$ & $p^{*}$ & $r$ & $p^{*}$ & $r$ & $p^{*}$ & $r$ & $p^{*}$ \\
\hline Age & -0.0127 & 0.8930 & 0.1691 & 0.0734 & 0.2202 & 0.0191 & 0.1902 & 0.0436 & 0.0945 & 0.3194 \\
\hline Gender & -0.0623 & 0.5120 & 0.1474 & 0.1191 & -0.0729 & 0.4429 & -0.0762 & 0.4223 & 0.1380 & 0.1448 \\
\hline Bodyweight & -0.0417 & 0.6683 & -0.2497 & 0.0092 & -0.1195 & 0.2182 & -0.0907 & 0.3507 & -0.1156 & 0.2336 \\
\hline BMI & -0.0504 & 0.6100 & -0.3675 & 0.0001 & -0.0453 & 0.6460 & -0.0796 & 0.4197 & -0.1695 & 0.0839 \\
\hline PMI & -0.1605 & 0.1070 & -0.0610 & 0.5425 & -0.1211 & 0.2252 & -0.0484 & 0.6293 & -0.0676 & 0.4998 \\
\hline AST & 0.1947 & 0.0388 & -0.2462 & 0.0086 & -0.0894 & 0.3464 & 0.0226 & 0.8120 & 0.3504 & 0.0001 \\
\hline ALT & 0.0521 & 0.5835 & -0.1792 & 0.0575 & -0.0647 & 0.4959 & -0.0707 & 0.4567 & 0.4015 & $\hat{0} .0001$ \\
\hline Total bilirubin & 0.1580 & 0.0947 & -0.3090 & 0.0009 & -0.1947 & 0.0388 & -0.0392 & 0.6804 & -0.0671 & 0.4800 \\
\hline Albumin & -0.3152 & 0.0007 & -0.0022 & 0.9819 & -0.1280 & 0.1766 & -0.4398 & $\begin{array}{l}< \\
0.0001\end{array}$ & -0.2312 & 0.0137 \\
\hline $\begin{array}{l}\text { Prothrombin } \\
\text { time }\end{array}$ & -0.1141 & 0.2311 & 0.2386 & 0.0113 & -0.1582 & 0.0957 & -0.0752 & 0.4308 & -0.0918 & 0.3357 \\
\hline Creatinine & 0.2197 & 0.0199 & 0.4322 & $\hat{0.0001}$ & 0.5714 & $\hat{0.0001}$ & 0.4085 & $\hat{0} .0001$ & 0.3497 & 0.0001 \\
\hline BUN & 0.2575 & 0.0059 & 0.5037 & $\hat{0.0001}$ & 0.5074 & $\hat{0.0001}$ & 0.3703 & $\hat{0} .0001$ & 0.4430 & $\begin{array}{l}< \\
0.0001\end{array}$ \\
\hline eGFR & -0.2490 & 0.0078 & -0.3451 & 0.0002 & -0.6086 & $\hat{0} .0001$ & -0.4481 & $\hat{0} .0001$ & -0.2788 & 0.0028 \\
\hline Serum sodium & -0.1200 & 0.2181 & -0.1232 & 0.2062 & -0.0285 & 0.7711 & -0.2553 & 0.0080 & -0.1790 & 0.0650 \\
\hline CRP & 0.2701 & 0.0047 & 0.3677 & $<0.0001$ & 0.0518 & 0.5944 & 0.4291 & $\begin{array}{l}<.0001 \\
0 .\end{array}$ & 0.2281 & 0.0176 \\
\hline Copeptin & - & - & 0.1969 & 0.0366 & 0.3104 & 0.0008 & 0.3182 & 0.0006 & 0.1709 & 0.0703 \\
\hline ZAG & 0.1969 & 0.0366 & - & - & 0.2701 & 0.0038 & 0.3634 & $\hat{0} .0001$ & 0.1735 & 0.0662 \\
\hline Cystatin C & 0.3104 & 0.0008 & 0.2701 & 0.0038 & - & - & 0.4913 & $\hat{0} .0001$ & 0.4468 & $\begin{array}{l}< \\
0.0001\end{array}$ \\
\hline NGAL & 0.3182 & 0.0006 & 0.3634 & $\hat{0} .0001$ & 0.4913 & $\begin{array}{l}< \\
0.0001\end{array}$ & - & - & 0.3105 & 0.0008 \\
\hline L-FABP & 0.1709 & 0.0703 & 0.1735 & 0.0662 & 0.4468 & $\begin{array}{l}<.0001 \\
0.0\end{array}$ & 0.3105 & 0.0008 & - & - \\
\hline Child-Pugh score & 0.3819 & $<0.0001$ & -0.0271 & 0.7754 & 0.1468 & 0.1208 & 0.3876 & $\hat{0} .0001$ & 0.2143 & 0.0227 \\
\hline ALBI score & 0.3366 & 0.0003 & -0.0921 & 0.3322 & 0.0656 & 0.4901 & 0.3750 & $\hat{0} .0001$ & 0.2078 & 0.0272 \\
\hline MELD score & 0.3057 & 0.0010 & 0.3338 & 0.0003 & 0.5595 & $\begin{array}{l}<.0001 \\
0.0\end{array}$ & 0.4498 & $\hat{0} .0001$ & 0.2839 & 0.0024 \\
\hline
\end{tabular}

*Spearman's rank correlation coefficient. BMI, body mass index; PMI, psoas muscle index; AST, aspartate aminotransferase; ALT, alanine aminotransferase; BUN, blood urea nitrogen; eGFR, estimated glomerular filtration rate; CRP, C-reactive protein; ZAG, zinc-a2- 
glycoprotein; NGAL, neutrophil gelatinase-associated lipocalin; L-FABP, Liver-type fatty acid-binding protein; ALBI, albumin-bilirubin; MELD, Model for End-Stage Liver Disease.

Table 3. Characteristics of patients treated with Tolvaptan.

\begin{tabular}{|c|c|c|c|c|c|c|c|}
\hline \multirow[t]{2}{*}{ Parameters } & Responders & $\begin{array}{l}\text { Non- } \\
\text { responders }\end{array}$ & $p^{\dagger}$ & cutoff & ROCAUC & Sensitivity & Specificity \\
\hline & $\mathrm{n}=19$ & $\mathrm{n}=19$ & & & & & \\
\hline Age (years) & $66.9 \pm 10.4$ & $67.3 \pm 8.9$ & 0.7589 & - & - & - & - \\
\hline Gender, Male : Female & $13: 6$ & $11: 8$ & 0.5795 & - & - & - & - \\
\hline Bodyweight (kg) & $59.6 \pm 9.9$ & $57.3 \pm 9.3$ & 0.4229 & - & - & - & - \\
\hline Body mass index $\left(\mathrm{kg} / \mathrm{m}^{2}\right)$ & $22.9 \pm 4.1$ & $22.4 \pm 3.7$ & 0.8315 & - & - & - & - \\
\hline Psoas muscle index $\left(\mathrm{cm}^{2} / \mathrm{m}^{2}\right)$ & $5.7 \pm 1.4$ & $5.2 \pm 1.2$ & 0.5534 & - & - & - & - \\
\hline $\mathrm{HCV} / \mathrm{HBV} / \mathrm{HCV}+\mathrm{AL} / \mathrm{AL} / \mathrm{NBNC} /$ others & $7 / 0 / 2 / 6 / 2 / 2$ & $5 / 4 / 1 / 4 / 4 / 1$ & - & - & - & - & - \\
\hline presence of HCC, yes: no & $11: 8$ & $12: 7$ & 0.7862 & - & - & - & - \\
\hline \multicolumn{8}{|l|}{ Dose of diuretic drugs } \\
\hline Tolvaptan (3.75 mg/7.5 mg) & $15 / 4$ & $17 / 2$ & 0.6976 & - & - & - & - \\
\hline Furosemide (mg) & $31.4 \pm 18.5$ & $31.3 \pm 18.3$ & 0.8217 & - & - & - & - \\
\hline Spironolactone (mg) & $45.8 \pm 19.1$ & $39.7 \pm 19.3$ & 0.2548 & - & - & - & - \\
\hline Albumin (g/dL) & $3.0 \pm 0.5$ & $2.8 \pm 0.5$ & 0.8490 & - & - & - & - \\
\hline Total Bilirubin (mg/dL) & $3.2 \pm 4.2$ & $1.8 \pm 1.4$ & 0.1361 & - & - & - & - \\
\hline Child-Pugh score & $9.2 \pm 1.9$ & $8.6 \pm 1.5$ & 0.3679 & - & - & - & - \\
\hline ALBI score & $-1.5 \pm 0.5$ & $-1.5 \pm 0.5$ & 0.7371 & - & - & - & - \\
\hline FIB4-index & $9.3 \pm 0.5$ & $8.7 \pm 4.0$ & 0.9534 & - & - & - & - \\
\hline MELD score & $17.1 \pm 2.6$ & $18.2 \pm 4.2$ & 0.3655 & - & - & - & - \\
\hline Creatinine (mg/dL) & $0.8 \pm 0.3$ & $1.0 \pm 0.5$ & 0.2932 & - & - & - & - \\
\hline BUN (mg/dL) & $15.2 \pm 6.9$ & $22.9 \pm 6.5$ & 0.0014 & 18.3 & 0.8047 & 79 & 74 \\
\hline eGFR (mL/min/1.73m²) & $73.1 \pm 29.0$ & $62.3 \pm 27.5$ & 0.2093 & - & - & - & - \\
\hline Serum sodium (mEq/L) & $133.9 \pm 3.9$ & $135.4 \pm 4.7$ & 0.2909 & - & - & - & - \\
\hline CRP (mg/dL) & $2.1 \pm 2.6$ & $2.6 \pm 2.6$ & 0.5018 & - & - & - & - \\
\hline Copeptin (pmol/L) & $12.5 \pm 17.0$ & $24.4 \pm 26.4$ & 0.0265 & 10.1 & 0.7119 & 68 & 74 \\
\hline $\mathrm{ZAG}(\mu \mathrm{g} / \mathrm{mL})$ & $30.7 \pm 11.8$ & $37.9 \pm 9.6$ & 0.0142 & 32.4 & 0.7341 & 79 & 74 \\
\hline NGAL (ng/mL) & $123.7 \pm 106.4$ & $112.6 \pm 79.0$ & 1.0000 & - & - & - & - \\
\hline Cystatin C (mg/dL) & $1.1 \pm 0.3$ & $1.2 \pm 0.5$ & 0.9534 & - & - & - & - \\
\hline L-FABP (ng/mL) & $11.7 \pm 10.7$ & $14.9 \pm 10.9$ & 0.2429 & - & - & - & - \\
\hline
\end{tabular}

${ }^{\dagger}$ Mann-Whitney Utest, ROCAUC, receiver operating characteristic area under the curve. HCV, hepatitis C virus; HBV, hepatitis B virus; AL, alcohol; NBNC, non HCV non HBV; HCC, hepatocellular carcinoma; ALBI, albumin-bilirubin; FIB4-index, fibrosis-4; MELD, Model for End-Stage Liver Disease; BUN, blood urea nitrogen; eGFR, estimated glomerular filtration rate; CRP, C-reactive protein; ZAG, zinc-a2- 
glycoprotein; NGAL, neutrophil gelatinase-associated lipocalin; L-FABP, Liver-type fatty acid-binding protein. Responders were defined as decompensated liver cirrhosis patients in whom tolvaptan was an effective treatment for ascites.

Table 4. Predictors contributing to the effect of Tolvaptan in the treatment for ascites

\begin{tabular}{|lllllll|}
\hline Predictors & \multicolumn{2}{l}{ Univariate analysis } & \multicolumn{4}{c|}{ Multivariate analysis } \\
& OR & $95 \% \mathrm{Cl}$ & $p^{\S}$ & OR & $95 \% \mathrm{Cl}$ & $p^{\S}$ \\
\hline BUN & 10.50 & $2.54 \rightarrow 53.39$ & 0.0009 & 7.43 & $1.20 \rightarrow 64.69$ & $\mathbf{0 . 0 3 0 6}$ \\
Copeptin & 6.07 & $1.57 \rightarrow 27.02$ & 0.0083 & 9.12 & $1.54 \rightarrow 91.14$ & $\mathbf{0 . 0 1 3 6}$ \\
\hline ZAG & 10.50 & $2.54 \rightarrow 53.39$ & 0.0009 & 7.43 & $1.20 \rightarrow 64.69$ & $\mathbf{0 . 0 3 0 6}$ \\
\hline
\end{tabular}

p§; Logistic regression analysis; OR, odds ratio; $95 \% \mathrm{Cl}, 95 \%$ confidence interval

BUN, blood urea nitrogen; ZAG, zinc-a2-glycoprotein.

\section{Figures}

Figure 1
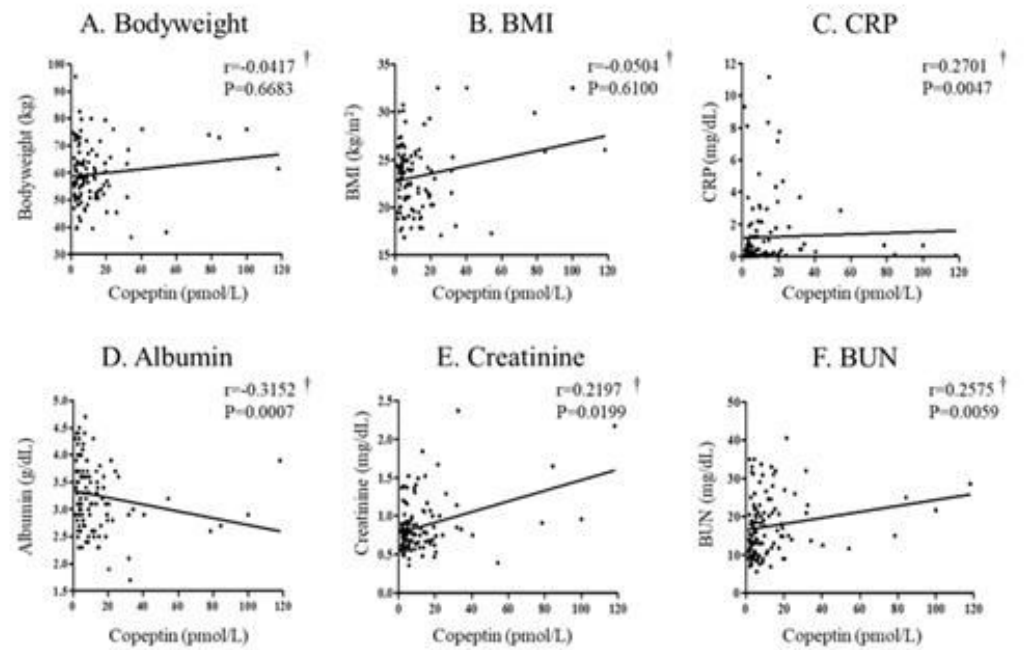

G. Bodyweight
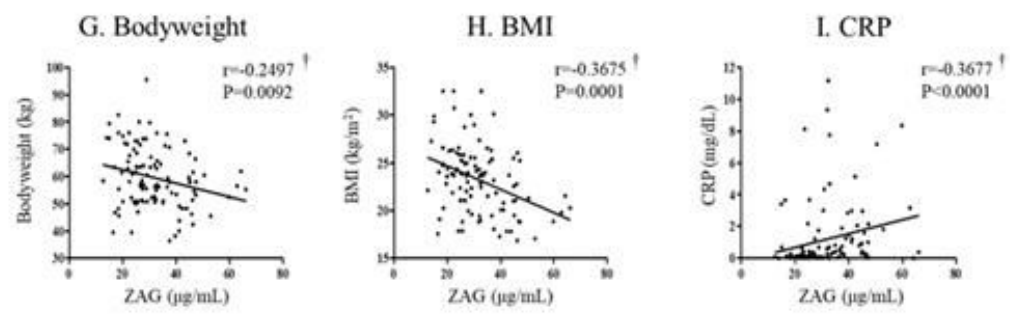

J. Albumin
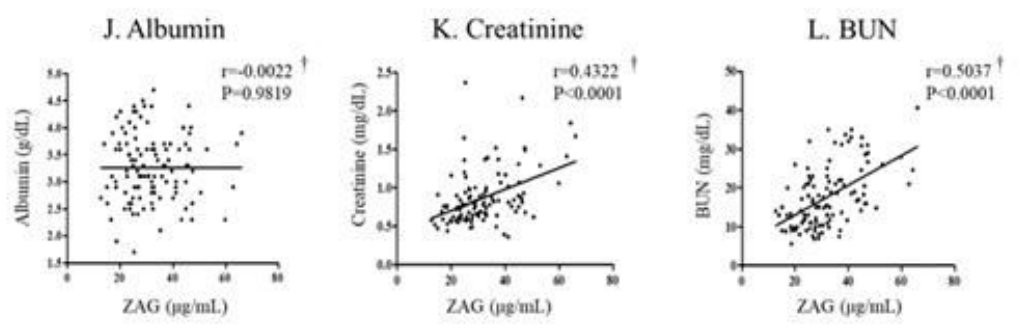

Figure 1

Serum copeptin levels were correlated with liver and kidney function in patients with cirrhosis. Serum ZAG levels were correlated with bodyweight, BMI and kidney function in patients with cirrhosis. Correlation between serum copeptin levels and Bodyweight (A), BMI 
(B), CRP (C), albumin (D), creatinine (E) or BUN (F). Correlation between serum ZAG levels and Bodyweight (G), BMI (H), CRP (I), albumin $(\mathrm{J})$, creatinine $(\mathrm{K})$ or BUN $(\mathrm{L})$. ZAG, zinc-a2-glycoprotein; BMI, body mass index; CRP, C-reactive protein; BUN, blood urea nitrogen

Figure 2
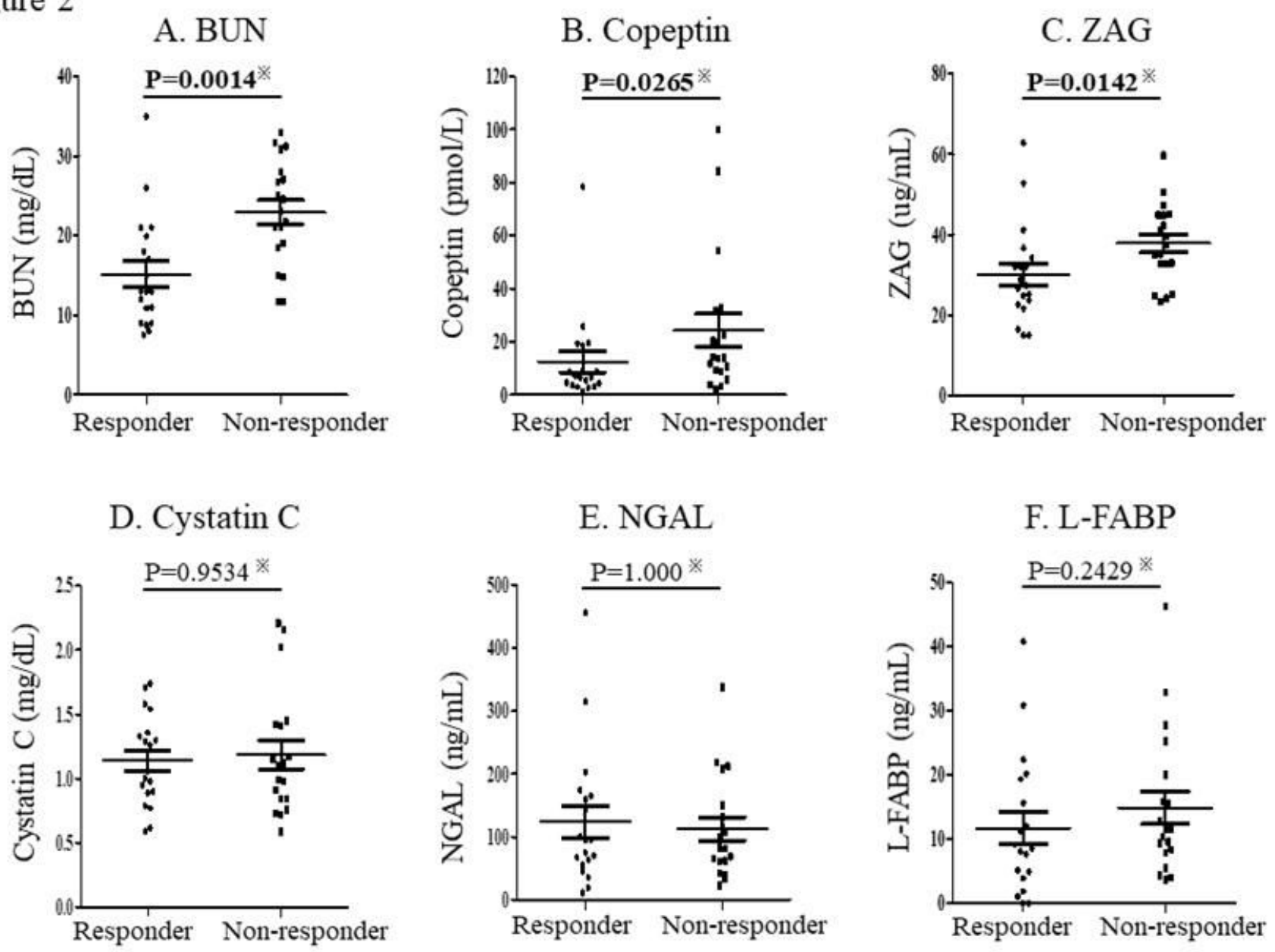

\section{Figure 2}

Predictors contributing to the effect of tolvaptan in treatment for ascites. Serum BUN (A), copeptin (B), ZAG (C), cystatin C (D), NGAL $(E)$ and L-FABL (F) levels in the Responder and Non-responder to tolvaptan. BUN, blood urea nitrogen; ZAG, zinc-a2-glycoprotein; NGAL, neutrophil gelatinase-associated lipocalin; L-FABP, liver-type fatty acid-binding protein

\section{Supplementary Files}

This is a list of supplementary files associated with this preprint. Click to download.

- SupplFig1.patientsflow.TIF 